\title{
Molybdenum sound velocity and shear modulus softening under shock compression
}

\author{
Jeffrey H. Nguyen, ${ }^{1,{ }^{*}}$ Minta C. Akin, ${ }^{1}$ Ricky Chau, ${ }^{1}$ Dayne E. Fratanduono, ${ }^{1}$ W. Patrick Ambrose, ${ }^{1}$ Oleg V. Fat'yanov, ${ }^{2}$ \\ Paul D. Asimow, ${ }^{2}$ and Neil C. Holmes ${ }^{1}$ \\ ${ }^{1}$ Lawrence Livermore National Laboratory, Livermore, California 94551, USA \\ ${ }^{2}$ California Institute of Technology, Pasadena, California 91125, USA
}

(Received 30 August 2013; revised manuscript received 1 May 2014; published 21 May 2014)

\begin{abstract}
We measured the longitudinal sound velocity in Mo shock compressed up to 4.4 Mbars on the Hugoniot. Its sound speed increases linearly with pressure up to $2.6 \mathrm{Mbars}$; the slope then decreases up to the melting pressure of $\sim 3.8$ Mbars. This suggests a decrease of shear modulus before the melt. A linear extrapolation of our data to 1 bar agrees with the ambient sound speed. The results suggest that Mo remains in the bcc phase on the Hugoniot up to the melting pressure. There is no statistically significant evidence for a previously reported bcc $\rightarrow$ hcp phase transition on the Hugoniot.
\end{abstract}

DOI: 10.1103/PhysRevB.89.174109

PACS number(s): 64.70.K-, 61.50.Ks, 64.70.dj, 81.30.-t

\section{INTRODUCTION}

Interest in complete equations of state and phase diagrams for transition metals such as $\mathrm{Ta}, \mathrm{Fe}, \mathrm{Cu}$, and $\mathrm{Mo}$ has recently surged. These metals have high melting temperatures at elevated pressures $(P)$ and are stable to high $P$ in diamond anvil cell (DAC) experiments, making them model candidates for studies at extreme conditions. To this end, many experiments and calculations have been published that address the high $P$ melt curves and existence of high $P$ phases [1-23].

These findings are controversial and often contradictory. The reports of "flat" melt curves for these metals $[1,2]$ are several thousand degrees below those previously reported [3$6,8,9]$ and disagree with melting predicted by the Lindemann criterion [24]. DAC studies show that Mo remains bec up to 5.6 Mbars at room temperature [9]. Hixson et al. [3] reported a bcc $\rightarrow$ hcp crystal structure transition at 2.1 Mbars $(\approx 4100 \mathrm{~K})$ and $\mathrm{hcp} \rightarrow$ liquid at 3.9 Mbars $(\approx 10000 \mathrm{~K})$ in their measurements of longitudinal sound speed $\left(C_{L}\right)$ in shock-compressed Mo. However, similar work in Fe showed that such a solid $\rightarrow$ solid transition depended on sample purity $[7,8]$. No such solid $\rightarrow$ solid transition is observed in Ta [6] or $\mathrm{Cu}[25]$ sound speed data. Theoretical studies disagree on the relative stability of the bcc and fcc phases $[17,21]$ or require the experimentally observed solid $\rightarrow$ liquid transition $[3,4]$ to be ignored or reinterpreted [15].

High $P$ DAC crystallographic experiments on Mo are restricted to room temperature [9], or to $\sim 1 \mathrm{Mbar}$ at high temperature $(T)$ [1,2], so the Hixson et al. [3] study anchors the high $P, T$ work. However, extrapolation of their results to ambient conditions disagrees with the measured ambient $C_{L}$ by $\sim 2 \mathrm{~km} / \mathrm{s}$. If correct, this would suggest a phase transition at $P<1$ Mbar, which has neither been predicted nor observed. This discrepancy between observed and extrapolated sound speed values may have arisen from the use of asymmetric impactors, which increases the uncertainty in $C_{L}$ by incorporating the uncertainties in the impactor Hugoniot and sound velocities. While the recent work by Kleiser et al. [10] appears to confirm Hixson et al.'s [3] results, the experimental design is such that it would be unable to detect faster $C_{L}$ that

*nguyen29@1lnl.gov would contradict those results. It is therefore prudent to repeat the experiment using the latest technology and symmetric impactors to confirm or reject the existence of the reported phase transitions.

\section{METHODS}

The longitudinal sound velocity $C_{L}$ is related to material properties by

$$
C_{L}=\left(\frac{K}{\rho}+\frac{4}{3} \frac{G}{\rho}\right)^{1 / 2},
$$

where $K$ and $G$ are the bulk and shear moduli, respectively, and $\rho$ is the material density. $C_{L}$ is sensitive to changes in crystal structure since either or both $K$ and $G$ change with phase. Indeed, $C_{L}$ measurements are used to determine melting on the Hugoniot since $G \approx 0$ in the fluid [3,6-8].

The experiment is based on the idea that the velocity of a rarefaction wave is faster than the initial shock wave in the laboratory frame [26-28]. Since $U_{s}<C_{L}+U_{p}$, the rarefaction wave eventually overtakes the shock front. Both mass velocity $U_{p}$ and shock velocity $U_{s}$ are known from previous experiments [4].

We need to determine the sample thickness $D$ required for a rarefaction wave moving at $C_{L}+U_{p}$ to overtake the shock front (Fig. 1) in Mo. Since we cannot directly observe the rarefaction wave catching up to the shock wave inside the metal, we observe this catchup in bromoform $\left(\mathrm{CHBr}_{3}\right)$, the analyzer fluid, in contact with the sample. In Fig. $1 \mathrm{CHBr}_{3}$ fills the region between the sample and window. When the shock enters the $\mathrm{CHBr}_{3}$, the $\mathrm{CHBr}_{3}$ emits light at an intensity proportional to $U_{p, \mathrm{Br}}^{7.6}$ [29], where $U_{p, \mathrm{Br}}$ is the $\mathrm{CHBr}_{3}$ mass velocity (Fig. 2). This provides a sharp increase in emission upon shock breakout and abruptly decreases when the release catches up to the shock front and weakens it. The time interval between the onset of emission and the abrupt decrease $\Delta t$ depends upon the thickness of Mo transited. This idea was used in previous sound speed experiments $[3,6-8,26]$. Because the analyzer has a lower shock impedance $\left(\rho U_{s}\right)$ than the Mo sample, a backwards-going rarefaction wave is formed when the shock arrives at the sample/analyzer interface, labeled as "R" in Fig. 1. That rarefaction wave perturbs the velocity of the overtaking wave used to determine sound velocity. The 


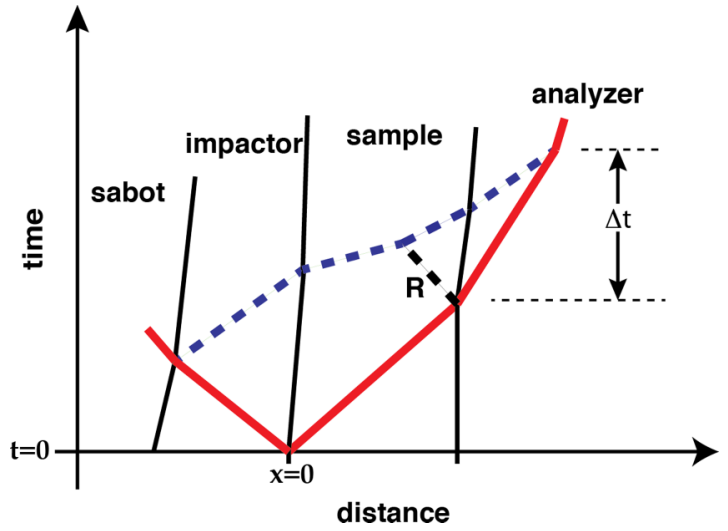

FIG. 1. (Color online) $x$ - $t$ diagram showing a rarefaction wave moving at $C_{L}+U_{p}$ (dashed blue line) overtaking the shock wave moving at $U_{s}$ (solid red line) in the sample and analyzer in the laboratory rest frame. Bold black lines show the position of the interfaces between the impactor and sample target layers. Projectile impact on the target occurs at $(x, t)=(0,0)$. The time interval between the arrival of the shock at the sample-analyzer interface and the overtake of the shock by the rarefaction wave is $\Delta t$. The rarefaction labeled $\mathrm{R}$ arises from the shock impedance mismatch between the sample and analyzer.

experiment is designed so that we can determine the value of $D$ for which the catchup rarefaction and the shock arrive at the sample/analyzer interface simultaneously $(\Delta t=0)$ for each experiment, and minimize its uncertainty.

From $D$ we can determine the sound speed at the shock pressure,

$$
C_{L}=\frac{\rho_{0}}{\rho} \frac{D+d}{D-d} U_{s}
$$

where $\rho_{o}$ and $\rho$ are initial and shocked densities, $d$ is the impactor thickness, and $U_{s}$ is shock speed in Mo.

The main source of uncertainty in $C_{L}$ is the experimental determination of $D$. The value of $\Delta t$ decreases linearly as the Mo sample thickness approaches $D$. We use a sample with varying Mo thickness, and find $D$ by extrapolation of the measured catchup times to $\Delta t=0$. To improve accuracy we use six thickness (Fig. 3) and use sample plates nearly as thick as $D$ to minimize the extrapolation distance. Using six target steps requires extensive two-dimensional hydrodynamic simulations. These were carried out at various impactor velocities to ensure one-dimensional hydrodynamic flow in the observation area prior to the catchup event.

A two-stage light-gas gun launched 0.8-mm-thick impactors at the target [30]. At impact, two shock waves are generated, one into the stepped sample, the other into the impactor (solid red lines in Fig. 1). The shock moves through each target step into the $\mathrm{CHBr}_{3}$, which emits light. Meanwhile, the reverse-launched shock into the impactor traverses the impactor thickness, where it arrives at the Mo/Lexan interface of the impactor, and launches a release wave. The head of the release wave (dashed blue line in Fig. 1) travels toward the $\mathrm{CHBr}_{3}$ through the compressed Mo impactor and target at the local Mo sound speed $C_{L}$.

The target and impactors were $99.99 \%$ pure Mo purchased from ESPI Metals. To achieve the highest pressure (4.4 Mbars),

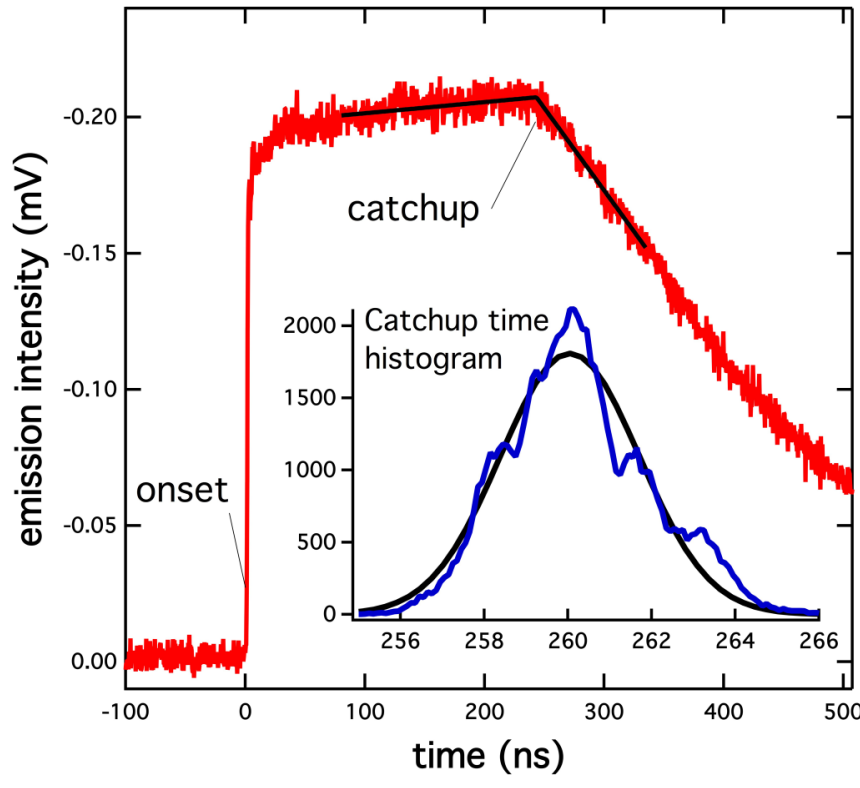

FIG. 2. (Color online) Typical $\mathrm{CHBr}_{3}$ emission. Intensity of emitted light is measured as a negative value. At the arrival of the shock front at the $\mathrm{Mo}-\mathrm{CHBr}_{3}$ interface, a rapid change in mass velocity $U_{p}$ produces a correspondingly rapid rise in the intensity of light in the $\mathrm{CHBr}_{3}$ analyzer ("onset"). Once the sound wave catches up to the shock wave ("catchup"), the signal decreases. Inset: The calculated catchup times from the randomly sampled fits are plotted as a histogram (blue). The Gaussian fit to the histogram (black) is used to determine $\Delta t$ and $\sigma$ for each channel. $\Delta t$ and $\sigma$ are then used to extrapolate to $D$ as shown in Fig. 4 .

a Ta impactor was used instead of Mo. Targets comprise six countersunk pockets of varying thicknesses between 1.0 and $3.0 \mathrm{~mm}$, depicted in Fig. 3. The baseplate was assembled in a liquid-tight container. We filled the detector side of the baseplate with degassed $\mathrm{CHBr}_{3}$. A fiber optic and lens assembly collected light at two distinct $\sim 200 \mu \mathrm{m}$ diameter spots behind each pocket, for a total of 12 measurements.

Onset is easily identified. To find the catchup time (Fig. 2), the intersection of two linear segments is calculated as described in Akin and Nguyen [31]. The primary source of uncertainty in $\Delta t$ and $C_{L}$ arises from this calculation. To determine this calculation's impact, we use a Monte Carlo sampling algorithm. The results are tabulated and fit to a

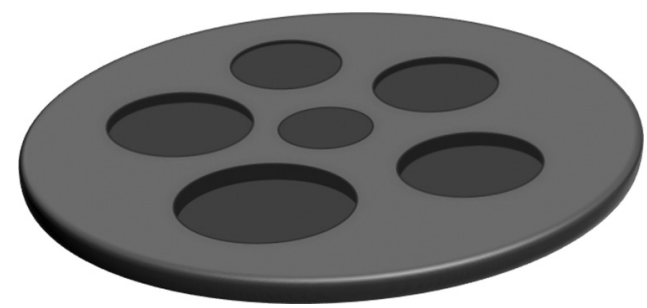

FIG. 3. The Mo baseplate has six pockets of varying depth. Positions of the pockets are optimized to remove side release effects in the data. Each pocket is imaged at two spots near the pocket center, with each spot roughly $200 \mu \mathrm{m}$ in diameter. The impactor approaches from the flat underside of the target, obscured in this view. The pocket diameters vary from 6 to $10 \mathrm{~mm}$. Baseplate diameter is $32 \mathrm{~mm}$. 


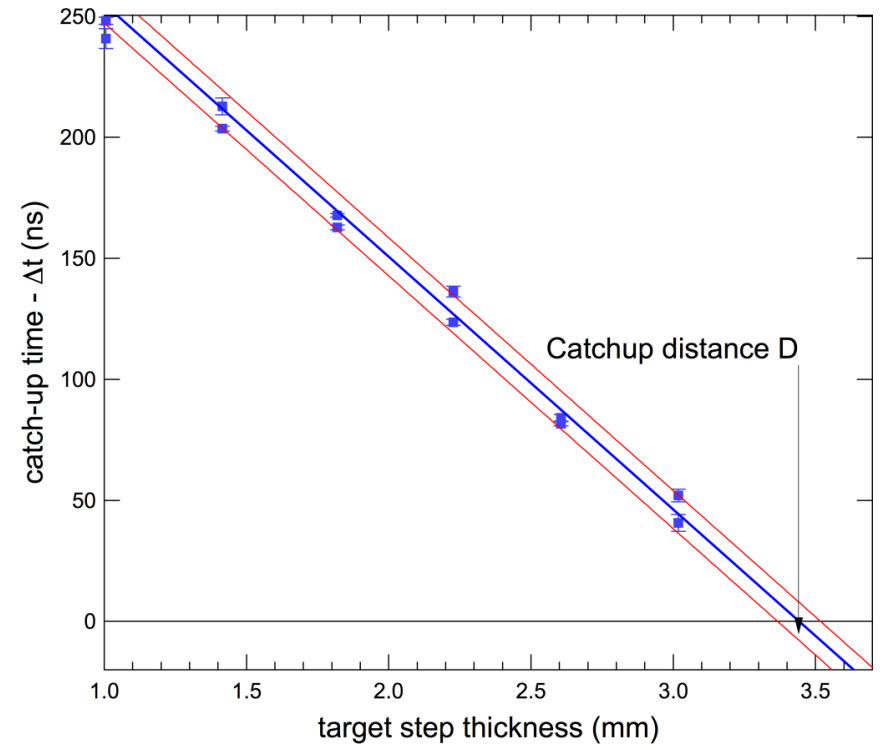

FIG. 4. (Color online) Catchup time $\Delta t$ as a function of Mo baseplate step thickness. Extrapolated to $\Delta t=0$, it yields the sample thickness at which catchup takes place at the Mo sample/analyzer boundary $D$.

Gaussian distribution (inset, Fig. 2). The mean and standard deviation of that Gaussian function are used as the catchup time and uncertainty $(\sigma)$ in finding $\Delta t$. Extrapolation of a $\sigma$-weighted linear fit to $\Delta t$ 's dependence on step height determines the catchup distance $D$ (Fig. 4). In cases where non-Gaussian character (e.g., a boxy or bimodal distribution) was seen, we chose a broader $\sigma$ Gaussian fit to overestimate the $\sigma$ used in extrapolation while capturing the mean sampled catchup time.

Equation (2) uses $D$ to find $C_{L}$ at the experimental pressure. Previous studies of the Mo Hugoniot [4] provided a straightforward way to determine $P, \rho$ and their uncertainties given only impactor velocities. Uncertainties in $D, \rho$, and the Mo Hugoniot are propagated to find the uncertainty of $C_{L}$, $\sigma_{C_{L}}$, typically $0.5 \%$ in this study.

\section{RESULTS AND DISCUSSION}

We plot the new $C_{L}$ data with those of Hixson et al. [3] in Fig. 5. New data are also listed in Table I. The Hixson data are further differentiated by impactor type for each shot. Over the range of $1.8<P<3.5 \mathrm{Mbars}$, the data agree within their respective uncertainties. The reader may observe the increased error bars above $300 \mathrm{GPa}$ on shots 450, 451, 4166, and 4168. The increased error on shots 450 and 4166 are due to attenuated light signals. On shot 450 this was due to a poorer quality (i.e., darker) batch of bromoform, which attenuates light signals. Data signals on shot 4166 were attenuated due to switching filters with a location change (Caltech to LLNL). Data signals on shot 4168 were not attenuated, but 4168 used a Ta impactor, which leads to a comparable increase in uncertainty. Shot 451's larger uncertainty is due to differences between channel-tochannel times on the two thicker steps. Masking these steps leads to calculated sound speeds of $9.65-9.85 \mathrm{~km} / \mathrm{s}$.

We note three significant differences between these new $C_{L}$ data and Hixson et al.'s [3]. First, model statistics do not

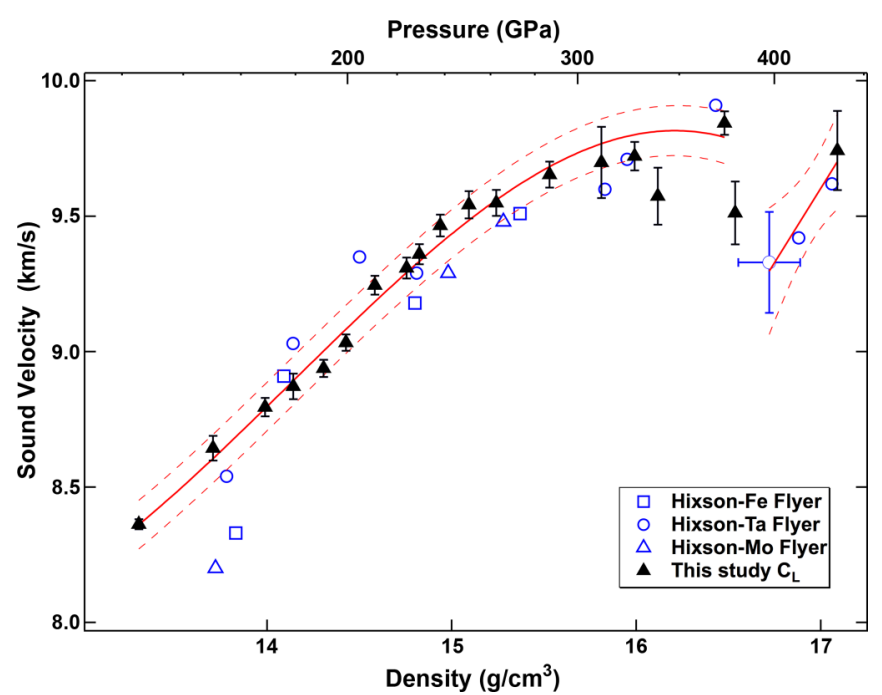

FIG. 5. (Color online) Mo sound speed as a function of $P$ and $\rho$. Solid symbols represent our data; open symbols are Hixson et al.'s data. The latter data are further differentiated by impactor used. Hixson's data error bars are nominally $2 \%$ [3], and are shown on only one data point for clarity. $1-\sigma$ prediction bands are included in a fit to our data below the melting pressure. A linear fit with Hixson's data [3] is also shown above the melting pressure. A single data point at $16.54 \mathrm{~g} / \mathrm{cc}$ was not used in either fit.

support a solid $\rightarrow$ solid phase transition at 2.1 Mbars. Second, the $C_{L}$ vs $\rho$ slope, for $P<2.6$ Mbars is significantly lower than that of Hixson et al. [3]; this pressure range includes the putative solid $\rightarrow$ solid transition. Third, $C_{L}$ ceases increasing linearly above $\sim 2.6$ Mbars. We will examine each of these points in detail.

To address the existence of a solid $\rightarrow$ solid phase transition at 2.1 Mbars [3], we proposed eight statistical models supporting a phase transition (two solid phases) and one model debunking this same phase transition (one solid phase). The models and associated statistical analyses are included in the Appendix below. The latter model follows Birch's law, a linear approximation to the sound speed equation $[32,33]$. This model agrees well with our data. The two-phase models also capture all the data. However, as argued in the Appendix, it is more likely that the two-phase models fit the noise in the data rather than the underlying physics. We therefore cannot reject a simpler model of no phase transition in favor of a two-phase system.

The discrepancy between our results and those of Hixson et al. [3] can be partly explained by their use of asymmetric impactors. In a symmetric impact experiment, Mo impactor on Mo sample $C_{L}$ can be determined from Eq. (2) where only the Mo Hugoniot shock speed $U_{S}$ and density $\rho$ are unmeasured. In nonsymmetric experiments, two additional variables from the impactor $C_{L}^{i}$ and $U_{S}^{i}$ must be used to find $C_{L}$, increasing uncertainties significantly. In Hixson et al.'s [3] data, the key point at the putative solid-solid phase transition (2.1 Mbars, or $14.6 \mathrm{~g} / \mathrm{cc}$ ) is from a nonsymmetric impact experiment.

Flat melt curve interpretations $[1,2,15]$ depend on the existence of a phase transition at 2.1 Mbars, and are inconsistent with the melt transition at 3.9 Mbars [3]. Extrapolation of Hixson's $C_{L}$ to ambient Mo $\rho_{0}$ results in a value much lower than the ambient $C_{L}$ of $6.25 \mathrm{~km} / \mathrm{s}$, due to the high $b$. This 
TABLE I. Experimental data as presented in Fig. 5.

\begin{tabular}{|c|c|c|c|c|c|c|c|}
\hline Shot & $\begin{array}{l}\text { Flyer velocity } \\
\qquad(\mathrm{km} / \mathrm{s})\end{array}$ & $\begin{array}{l}\text { Flyer thickness } \\
\qquad(\mathrm{mm})\end{array}$ & $\begin{array}{c}\text { Shock velocity } \\
(\mathrm{km} / \mathrm{s})\end{array}$ & $\begin{array}{l}\text { Density } \\
(\mathrm{g} / \mathrm{cc})\end{array}$ & $\begin{array}{l}\text { Pressure } \\
(\mathrm{GPa})\end{array}$ & $\begin{array}{l}\text { Catchup distance } \\
\qquad(\mathrm{mm})\end{array}$ & $\begin{array}{l}\text { Sound velocity } \\
(\mathrm{km} / \mathrm{s})\end{array}$ \\
\hline 465 & $3.3960(5)$ & $0.8102(4)$ & $7.273(10)$ & $13.3040(14)$ & $126.1(2)$ & $4.053(13)$ & $8.363(18)$ \\
\hline 464 & $3.876(1)$ & $0.8108(4)$ & $7.575(11)$ & $13.7070(16)$ & $149.8(2)$ & $3.85(4)$ & $8.64(5)$ \\
\hline 438 & $4.226(1)$ & $0.804(1)$ & $7.791(12)$ & $13.9880(16)$ & $168.1(3)$ & $3.74(3)$ & $8.80(3)$ \\
\hline 437 & $4.405(6)$ & $0.802(1)$ & $7.909(13)$ & $14.141(5)$ & $177.7(4)$ & $3.69(4)$ & $8.87(5)$ \\
\hline 422 & $4.614(6)$ & $0.793(1)$ & $8.031(14)$ & $14.306(5)$ & $189.2(5)$ & $3.620(18)$ & $8.94(3)$ \\
\hline 424 & $4.766(1)$ & $0.789(1)$ & $8.134(14)$ & $14.4270(16)$ & 197.7(4) & $3.553(17)$ & $9.03(3)$ \\
\hline 423 & $4.964(4)$ & $0.785(1)$ & $8.258(14)$ & $14.583(4)$ & 209.1(4) & $3.399(19)$ & $9.25(4)$ \\
\hline 426 & $5.184(1)$ & $0.793(1)$ & $8.396(15)$ & $14.7550(17)$ & $222.0(4)$ & $3.42(2)$ & $9.31(4)$ \\
\hline 425 & $5.273(3)$ & $0.779(1)$ & $8.452(15)$ & $14.824(3)$ & $227.3(5)$ & $3.33(2)$ & $9.36(4)$ \\
\hline 421 & $5.420(5)$ & $0.788(1)$ & $8.544(16)$ & $14.938(4)$ & $236.2(5)$ & $3.32(2)$ & $9.47(4)$ \\
\hline 443 & $5.622(13)$ & $0.786(1)$ & $8.671(18)$ & $15.093(10)$ & $248.6(9)$ & $3.29(2)$ & $9.54(5)$ \\
\hline 441 & $5.817(10)$ & $0.802(1)$ & $8.793(18)$ & $15.241(8)$ & $260.8(8)$ & $3.38(2)$ & $9.55(5)$ \\
\hline 477 & $6.201(5)$ & $0.8000(1)$ & $9.034(18)$ & $15.530(4)$ & $285.6(7)$ & $3.35(3)$ & $9.65(5)$ \\
\hline 450 & $6.582(1)$ & $0.806(1)$ & $9.273(19)$ & $15.8110(17)$ & $311.2(7)$ & $3.40(9)$ & $9.70(13)$ \\
\hline 480 & $6.829(5)$ & $0.8106(5)$ & $9.43(2)$ & $15.991(4)$ & $328.2(8)$ & $3.44(3)$ & $9.72(5)$ \\
\hline 451 & $7.002(3)$ & $0.806(1)$ & $9.54(3)$ & $16.12(2)$ & $340(2)$ & $3.56(6)$ & $9.57(10)$ \\
\hline 4167 & $7.513(2)$ & $0.8080(4)$ & $9.86(2)$ & $16.478(3)$ & $377.5(9)$ & $3.442(18)$ & $9.84(4)$ \\
\hline 4166 & $7.594(2)$ & $0.8112(4)$ & $9.91(2)$ & $16.535(3)$ & $383.5(9)$ & $3.73(9)$ & $9.51(12)$ \\
\hline 4168 & $7.625(2)$ & $0.786(1)^{\mathrm{a}}$ & $10.36(2)$ & $17.089(3)$ & $438(8)$ & $4.66(2)$ & $9.74(15)$ \\
\hline
\end{tabular}

${ }^{a}$ Shot 4168 was the only nonsymmetric shot in this study. Shot 4168 used a $0.8 \mathrm{~mm}$ Ta impactor to shock Mo well into the liquid phase. Error bars on shot 4168 are accordingly larger due to the additional uncertainties in the Ta Hugoniot. Shots 4166-4168 were done at LLNL, the rest were carried out at Caltech.

implies another solid $\rightarrow$ solid transition, and that the existing phase on the Hugoniot is not bcc at $P$ immediately below 2.1 Mbars. Alternately, pressure-induced $s \rightarrow d$ electronic transfer was considered the cause of this anomalous behavior $[3,34]$. Linear extrapolation of the new data (with the lower $b$ ) to ambient yields $C_{L}=6.3 \pm 0.15 \mathrm{~km} / \mathrm{s}$. This is consistent with the hypothesis that Mo remains in the bcc phase at $P \lesssim 2.6$ Mbars, and does not support a flat melt curve interpretation. This result is supported by preliminary $\mathrm{x}$-ray diffraction work showing Mo is bcc at $~ 3$ Mbars [35].

Above $\sim 2.9$ Mbars, these data suggest a decreasing shear modulus in Mo, shown by a decrease in $b$ from 2.9 Mbars to the melt curve. This behavior was seen in $\mathrm{Cu}$ sound speed experiments [25], and is consistent with a predicted decrease of shear modulus prior to melt. Shear-wave velocity in the Earth's inner core also decreases near the melting pressuretemperature. Martorell et al. [36] attributed this decrease in elastic properties of hcp-Fe. For Mo in particular, our sound speed results are consistent with calculations by Cazorla et al. [17] showing that the bcc phase is more stable than the fcc phase up to 3.5 Mbars.

\section{CONCLUSIONS}

We measured the longitudinal sound speed $C_{L}$ of Mo shocked to 1.3 to 4.0 Mbars using symmetric impacts. A single nonsymmetric impactor was used to shock Mo to 4.4 Mbars. The rate of change of sound speed with density is lower than that observed by Hixson et al. [3] and extrapolates to the measured ambient $C_{L}$. It is consistent with a stable bcc phase to at least 3.4 Mbars. We see no evidence for a previously reported solid-solid phase transition at 2.1 Mbars $[3,10]$. Instead, these data show a stable phase up to 3.4 Mbars, with decrease of the shear modulus starting as early as 2.6 Mbars.

\section{ACKNOWLEDGMENTS}

We thank R. Hixson and M. Ross for their useful discussions, and Papo Gelle, Mike Long, Russ Oliver, Bob Nafzinger, Paul Benevento, Sam Weaver, and Cory McLean for their dedicated effort. Lawrence Livermore National Laboratory is operated by Lawrence Livermore National Security, LLC, for the U.S. Department of Energy, National Nuclear Security Administration under Contract DE-AC52-07NA27344.

\section{APPENDIX: STATISTICAL ANALYSIS}

Since previous work by Hixson et al. [3] suggested a solidsolid phase transition at 2.1 Mbars, we fitted our sound speed data from 1.26 to 2.60 Mbars to single-phase models and twophase models. The single-phase model follows Birch's law, a linear approximation to the sound speed equations derived from lattice dynamics [32,33],

$$
C_{L}=a+b \rho,
$$

where $a$ and $b$ are constants for a phase. Similarly, the twophase model consists of two lines, one before and one after the phase transition,

$$
C_{L}=\left(\begin{array}{ll}
a_{1}+b_{1} \rho & \rho<\rho_{\text {trans }} \\
a_{2}+b_{2} \rho & \rho>\rho_{\text {trans }}
\end{array}\right) .
$$

Strictly speaking, the one- and two-phase models cannot be treated as nested due to their different functional forms, and so tests such as the likelihood ratio, F-test, or $\chi^{2}$-difference tests are invalid. However, we can compare the relative quality of fit for these models to the single-phase model and make some estimates regarding the value of the more complicated model. 
TABLE II. Goodness-of-fit results from nine models of sound speed data up to $15.15 \mathrm{~g} / \mathrm{cc}$. Models 1-8 assume a two-phase model with the phase transition occurring at $\rho_{\text {trans }} \cdot \chi^{2}$ values, degrees of freedom $(v)$, the probability of obtaining a larger $\chi^{2}\left(Q_{\chi^{2}, d}\right)$, and extrapolation of the model to calculated the sound speed at ambient condition $\left(C_{L, 0}\right)$ are shown on the right.

\begin{tabular}{lccccc}
\hline \hline Model & $\rho_{\text {trans }}$ & $\chi^{2}$ & $v$ & $Q_{\chi^{2}, v}$ & $C_{L, 0}$ \\
\hline two-phase & & & & & \\
1 & 13.85 & 8.60 & 9 & 0.475 & 6.22 \\
2 & 14.06 & 6.26 & 9 & 0.714 & 6.40 \\
3 & 14.22 & 5.76 & 9 & 0.763 & 6.44 \\
4 & 14.37 & 6.38 & 9 & 0.702 & 6.55 \\
5 & 14.51 & 2.78 & 9 & 0.972 & 6.55 \\
6 & 14.67 & 10.14 & 9 & 0.340 & 6.43 \\
7 & 14.79 & 10.84 & 9 & 0.287 & 6.40 \\
8 & 14.88 & 11.21 & 9 & 0.262 & 6.22 \\
single phase & $\mathrm{n} / \mathrm{a}$ & 13.18 & 10 & 0.214 & 6.38 \\
\hline \hline
\end{tabular}

We chose to do so through the $\chi^{2}$ statistic,

$$
\chi^{2}=\Sigma\left(\frac{C_{L, \text { obs }}-C_{L, \text { model }}}{\sigma_{\text {obs }}}\right)^{2} .
$$

We also calculated the probability $Q_{\chi^{2}, v}$ that a larger calculated $\chi^{2}$ for the fit would be observed due to chance,

$$
Q_{\chi^{2}, v}=\left[2^{v / 2} \Gamma\left(\frac{v}{2}\right)\right]^{-1} \int_{\chi^{2}}^{\infty}(t)^{\frac{v}{2}-1} e^{-\frac{t}{2}} d x .
$$

where $v$ is the number of degrees of freedom (data points independent variables) and $\Gamma(x)=\int_{0}^{\infty}(t)^{x-1} e^{-t} d t$. We tested this model for phase transition at different densities. The resulting $\chi^{2}$ and $Q_{\chi^{2}, v}$ values, as well as the calculated sound speed at ambient pressure $C_{L, 0}$, are tabulated in
Table II for each fit. One of the single-phase models is included for comparison.

All of the models adequately capture the data. We then apply both statistic and physical tests to determine the correct model for this set of data. From a statistical point of view, the additional fit parameter of the two-phase models will lead to improved $\chi^{2}$ values compared to the single-phase model, as is shown in Table II. Such additional fit parameters increase the ability to fit noise in the data as well as the underlying physics. Because the single-phase model is sufficient to explain and predict the results, indicating that the physics has been adequately modeled, the additional complexity of a two-phase model is not justified. A specific case of model 5 will be discussed below. From a physics point of view, the singlephase model agrees with Birch's law best on extrapolation to ambient sound velocity $C_{L, 0}$. We therefore select the singlephase model.

There is a large difference in the $\chi^{2}$ value of the single-phase model and model 5 (Table II), which corresponds to a phase transition near the putative transition at 2.1 Mbars. This model has an unusually small $\chi^{2}$ statistic, which may encourage some readers to accept it as the "correct" model, and to argue that a transition exists at this pressure as a result. If we assume that model 5 were a perfect model of the actual underlying physics of the transition, and we repeated the experiment with similar levels of precision, we would obtain a $\chi^{2}$ value this size less than $3 \%$ of the time. It is more likely that model 5 is fitting noise in the data, rather than underlying physics. Given the good agreement of the single-phase fit, we cannot reject the simpler hypothesis of a single phase in favor of a two-phase system. Additional sound speed data in the 14.1$14.7 \mathrm{~g} / \mathrm{cc}$ density range, of better than $0.1 \%$ accuracy and precision, would be needed to further resolve these models. These authors feel that even with the improved design and analysis techniques presented here, such data would need to be taken at impractically frequent spacings, that we prefer an alternate diagnostic method.
[1] D. Errandonea, B. Schwager, R. Ditz, C. Gessmann, R. Boehler, and M. Ross, Phys. Rev. B 63, 132104 (2001).

[2] D. Santamaria-Perez, M. Ross, D. Errandonea, G. D. Mukherjee, M. Mezouar, and R. Boehler, J. Chem. Phys. 130, 124509 (2009).

[3] R. S. Hixson, D. A. Boness, J. W. Shaner, and J. A. Moriarty, Phys. Rev. Lett. 62, 637 (1989).

[4] R. S. Hixson and J. N. Fritz, J. Appl. Phys. 71, 1721 (1992).

[5] A. C. Mitchell and W. J. Nellis, J. Appl. Phys. 52, 3363 (1981).

[6] J. M. Brown and J. W. Shaner, in Shock Waves in Condensed Matter-1983, edited by J. R. Asay, R. A. Graham, and G. K. Straub (Elsevier, New York, 1984), p. 91.

[7] J. M. Brown and R. G. McQueen, Geophys. Res. Lett. 7, 533 (1980).

[8] J. H. Nguyen and N. C. Holmes, Nature (London) 427, 339 (2004).

[9] Y. K. Vohra and A. L. Ruoff, Phys. Rev. B 42, 8651 (1990).

[10] G. J. Kleiser, L. C. Chhabildas, W. D. Reinhart, and W. W. Anderson, in Shock Compression of Condensed Matter-2011, edited by M. L. Elert, W. T. Buttler, J. P. Borg, J. L. Jourdan, and T. J. Vogler (American Institute of Physics, Melville, NY, 2012), p. 1517.

[11] D. Errandonea, Physica B 357, 356 (2005).

[12] D. Errandonea, M. Somayazulu, D. Hausermann, and H. K. Mao, J. Phys. Condens. Matter 15, 7635 (2003).

[13] S. P. Marsh, LASL Shock Hugoniot Data (University of California Press, Berkeley, 1980), p. 658.

[14] R. G. McQueen, S. P. Marsh, J. W. Taylor, J. N. Fritz, and W. J. Carter, High Pressure Impact Phenomena, edited by R. Kinslow (Academic, New York, 1970), p. 293.

[15] M. Ross, D. Errandonea, and R. Boehler, Phys. Rev. B 76, 184118 (2007).

[16] F. Jona and P. M. Marcus, J. Phys. Condens. Matter 17, 1049 (2005).

[17] C. Cazorla, D. Alfè, and M. J. Gillan, Phys. Rev. B 85, 064113 (2012).

[18] J. C. Cazorla, M. J. Gillan, S. Taioli, and D. Alfè, J. Chem. Phys. 126, 194502 (2007).

[19] C. J. Wu, P. Söderlind, J. N. Glosli, and J. E. Klepeis, Nat. Mater. 8, 223 (2009). 
[20] S. Japel, B. Schwager, R. Boehler, and M. Ross, Phys. Rev. Lett. 95, 167801 (2005).

[21] A. B. Belonoshko, S. I. Simak, A. E. Kochetov, B. Johansson, L. Burakovsky, and D. L. Preston, Phys. Rev. Lett. 92, 195701 (2004).

[22] J. A. Moriarty, Phys. Rev. B 45, 2004 (1992).

[23] C. E. Ragan, M. G. Silbert, and B. C. Diven, J. Appl. Phys. 48, 2860 (1977).

[24] F. A. Lindemann, Phys. Z., 11, 609 (1910).

[25] D. Hayes, R. S. Hixon, and R. G. McQueen, in Shock Compression of Condensed Matter-1999, edited by M. D. Furnish, L. C. Chhabildas, and R. S. Hixon (American Institute of Physics, New York, 2000), p. 483.

[26] R. G. McQueen, J. W. Hopson, and J. N. Fritz, Rev. Sci. Instrum. 53, 245 (1982).

[27] L. V. Al'tshuler, S. B. Kormer, M. I. Brazhnik, L. A. Vladimirov, M. P. Speranskaya, and A. I. Funtikov, Sov. Phys. JETP-USSR, 11, 766 (1960).
[28] G. R. Fowles, J. Appl. Phys., 31, 655 (1960).

[29] R. G. McQueen and D. G. Isaak, in Shock Compression of Condensed Matter-1989, edited by S. C. Schmidt, J. N. Johnson, and L. W. Davison (North-Holland, Amsterdam, Netherlands, 1990), p. 125.

[30] A. H. Jones, W. M. Isbell, and C. J. Maiden, J. Appl. Phys. 37, 3493 (1966).

[31] M. C. Akin and J. H. Nguyen (unpublished).

[32] D. L. Anderson, Geophys. J. R. Astron. Soc. 13, 9 (1967).

[33] T. J. Shankland and D. H. Chung, Phys. Earth Planet. Int. 8, 121 (1974).

[34] B. K. Godwal and R. Jeanloz, Phys. Rev. B 41, 7440 (1990).

[35] J. Wang, F. Coppari, R. F. Smith, J. H. Eggert, T. Boehly, G. Collins, and T. S. Duffy, AGU Fall Meeting abstract (2014).

[36] B. Martorell, L. Vocadlo, J. Brodholt, and I. G. Wood, Science 342, 466 (2013). 